\title{
ИССЛЕДОВАНИЕ РАСПРЕДЕЛЕНИЯ ПОГЛОЩЕННОЙ ДОЗЫ ПРИ ФОТОН-ЗАХВАТНОЙ ТЕРАПИИ С ИНТРАТУМОРАЛЬНЫМ ВВЕДЕНИЕМ ДОЗОПОВЫШАЮЩЕГО АГЕНТА В МЕЛАНОМЕ В16F10
}

\author{
Ю. А. Финогенова \\ Национальный медицинский исследовательский центр онкологии имени Н. Н. Блохина, Москва \\ Федеральный медицинский биосизический центр имени А. И. Бурназяна, Москва \\ ${ }^{3}$ Институт общей и неорганической химии имени Н. С. Курнакова, Москва \\ ${ }^{4}$ Российский национальный исследовательский медицинский университет имени Н. И. Пирогова, Москва \\ ${ }^{5}$ Национальный исследовательский технологический университет «МИСиС», Москва \\ ${ }^{6}$ Сколковский институт науки и технологий, Москва \\ Московский клинический научно-практический центр имени А. С. Логинова, Москва
}

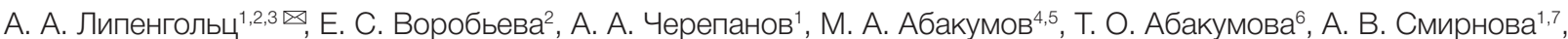

В фотон-захватной терапии (ФЗТ) величина поглощенной дозы определяется не только параметрами облучения, но и концентрацией дозоповышающего агента (ДПА) в облучаемом объекте. В данной работе было проведено расчетноэкспериментальное исследование распределения поглощенной дозы на опухолевой модели мышиной меланомы B16F10, после однократной интратуморальной инъекции висмута в качестве ДПА в форме водного раствора комплекса Ві-ДТПА. Оценку поглощенной дозы проводили для однофракционного рентгеновского облучения длительностью 28,5 мин. Количественное определение ДПА in vivo осуществляли при помощи микро-КТ, используя значения рентгеноплотности опухолевых тканей на полученных КТ-томограммах. В результате исследования установлено, что за счет присутствия ДПА в 6\% объема опухоли поглощенная доза увеличивалась более чем в 2 раза и в 29\% объема опухоли наблюдалось увеличение поглощенной дозы, отличное от 1. Время задержки роста опухоли, рассчитанное для полученного дозо-объемного распределения с учетом только непосредственного радиационного поражения опухолевых клеток, составило 0,76 суток, тогда как в ранее проведенных экспериментальных исследованиях данная величина равнялась 10 суткам. Полученное несоответствие может указывать на то, что торможение роста опухоли при ФЗТ с интратуморальным введением ДПА достигается за счет не только непосредственного радиационного поражения опухоли, но и иных противоопухолевых механизмов.

Ключевые слова: лучевая терапия, фотон-захватная терапия, меланома B16F10, увеличение дозы, КТ, дозообъемное распределение

Финансирование: исследование выполнено при финансовой поддержке гранта РНФ 18-13-00459.

$\triangle$ Для корреспонденции: Алексей Андреевич Липенгольц Каширское ш., д. 24, г. Москва, 115478; lipengolts@mail.ru

Статья получена: 28.09.2018 Статья принята к печати: 20.10.2018

DOI: $10.24075 /$ vrgmu.2018.062

\section{EVALUATION OF ABSORBED DOSE DISTRIBUTION IN MELANOMA B16F10 DURING CONTRAST ENHANCED RADIOTHERAPY WITH INTRATUMORAL ADMINISTRATION OF DOSE-ENHANCING AGENT}

Lipengolts $A A^{1,2,3 凶}$, Vorobyeva $E S^{2}$, Cherepanov $A A^{1}$, Abakumov $M A^{4,5}$, Abakumova $T^{6}$, Smirnova $A V^{1,7}$, Finogenova YuA ${ }^{4}$, Grigorieva $\mathrm{EYu}^{1,3}$, Sheino $\mathrm{IN}^{2}$, Kulakov VN ${ }^{2}$

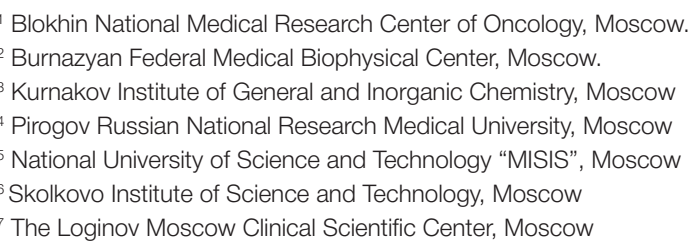

Contrast-enhanced radiotherapy (CERT) is a binary treatment modality in which the absorbed radiation dose is not only determined by the parameters of the external radiation source but also affected by the concentration of a dose-enhancing agent (DEA) in the studied object. In this work we assessed the distribution of the absorbed dose in a murine B16F10 melanoma injected with a single dose of an aqueous Bi-DTPA solution. The mice were exposed to a single fraction of X-ray irradiation for $28.5 \mathrm{~min}$. In vivo measurements of DEA concentrations were done on a micro-CT scanner using the radiopacity values of malignant tissues from the obtained CT images. We found that the presence of DEA enhanced the absorbed dose more than twofold in $6 \%$ of the tumor volume; in $29 \%$ of the tumor volume the absorbed dose increased more than onefold. The tumor growth delay time calculated for our model was 0.76 days (we only accounted for the damage caused directly by radiation), whereas in our previous research study tumor growth delay was 10 days. This discrepancy may indicate that in the tumors exposed to contrast-enhanced radiotherapy growth delay results from both the damage directly caused by radiation and other antitumor mechanisms.

Keywords: radiotherapy, contrast enhanced radiotherapy, melanoma B16F10, dose enhancement, CT, dose-volume histogram Funding: the study was supported by the Russian Science Foundation (Project ID 18-13-00459).

$\triangle$ Correspondence should be addressed: Alexey A. Lipengolts Kashirskoe shosse 24, Moscow, 115478; lipengolts@mail.ru

Received: 28.09.2018 Accepted: 20.10.2018

DOI: $10.24075 /$ brsmu.2018.062 
Борьба со злокачественными опухолями является важной задачей современного здравоохранения. Одним из методов лечения злокачественных новообразований является лучевая терапия. На сегодняшний день она успешно применяется при лечении многих видов рака, однако для ряда радиорезистентных опухолей ее эффективность все еще не превышает 30-50\% [1]. Повышение терапевтической эффективности лучевой терапии возможно за счет повышения поглощенной дозы в опухоли, однако лимитирующим фактором при этом становится лучевая нагрузка на нормальные ткани. Одним из методов, позволяющих локально увеличить поглощенную дозу в опухоли, без увеличения лучевой нагрузки на окружающие ткани, является фотон-захватная терапия (ФЗТ). Это увеличение дозы достигается путем введения или доставки в опухолевые ткани специальных препаратов, содержащих дозоповышающие агенты (ДПА). В ФЗТ к ним относятся химические элементы с порядковым номером Z более 52 (I, Gd, Au, Pt, Bi и др.). За счет большей вероятности взаимодействия таких элементов с внешним рентгеновским излучением удается достичь избирательного локального увеличения поглощенной дозы в области накопления препарата с ДПА [2-4]. В отличие от конвенциональной лучевой терапии, в которой используется геометрическое наведение излучения на опухоль, в ФЗТ возможна реализация биологического наведения излучения на мишень за счет использования туморотропных препаратов с ДПА. Противоопухолевая эффективность ФЗТ показана в ряде экспериментальных исследований на животных [5-11]. Получаемые в исследованиях результаты по терапевтической эффективности ФЗТ сложно транслировать в клиническую практику, так как без сопоставления наблюдаемого противоопухолевого эффекта с величиной и распределением поглощенной дозы в опухоли невозможно прогнозировать терапевтический исход.

Дозиметрическое сопровождение представляет собой наиболее сложный и наименее разработанный компонент ФЗТ как в экспериментальных исследованиях, так и в клинических. ФЗТ - бинарная технология лучевой терапии, в которой величина и пространственное распределение поглощенной дозы в мишени определяются не только параметрами внешнего облучения, но и концентрацией, а также пространственным распределением препарата с (ДПА).

Интратуморальное (внутриопухолевое) введение ДПА используется как при экспериментальных исследованиях противоопухолевой эффективности ФЗТ на лабораторных животных [12-17], так и в клинической практике [18].

Целью работы было провести количественное исследование пространственного распределения висмута в качестве ДПА для ФЗТ на примере меланомы B16F10 при однократном интратуморальном введении и оценить результаты дозо-объемного распределения.

\section{МАТЕРИАЛЫ И МЕТОДЫ}

Исследование проводили на самках мышей линии C57BI/6 массой 20-22 г (питомник филиала "Столбовая" ФГБУН НЦБТ ФМБА; Россия). Животных содержали при естественном освещении в стандартных условиях конвенционального вивария. В качестве опухолевой модели использовали мышиную меланому B16F10. Опухоль перевивали подкожно на среднюю треть латеральной части правой задней лапы. Перевивку осуществляли 14\%-й суспензией опухолевого материала в объеме 0,2 мл среды Хенкса ex tempore. Исследование распределения ДПА в опухолях шести животных проводили по достижении

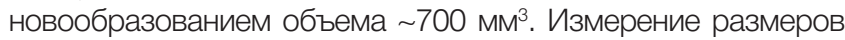
опухолевого узла проводили штангенциркулем в трех взаимно перпендикулярных плоскостях с последующим вычислением объема опухоли по формуле для вычисления объема эллипсоида.

В качестве ДПА применяли висмут в виде комплекса с диэтилентриаминопентауксусной кислотой (ВiДТПА), использованный нами ранее в исследованиях противоопухолевой эффективности [15]. Характеристика использованного раствора Ві-ДТПА представлена в таблице.

Введение ДПА осуществляли однократной интратуморальной инъекцией 50 мкл раствора Ві-ДТПА, содержащего 5 мг висмута.

Количественное исследование распределения ДПА in vivo проводили методом микро-КT [19-21] на сканере IVIS Spectrum CT (Perkin Elmer; США). Во время исследования мыши находились в состоянии общей анестезии, которую осуществляли ингаляционной подачей воздушной смеси с 2\%-м объемным содержанием изофлюрана. Сканирование всего тела мышей проводили до введения ДПА, сразу же после введения ДПА ( 1 мин после введения) и далее через 3, 5, 10, 15, 20, 25 и 30 мин. Пример томограмм опухолей до и после интратуморального введения ДПА представлен на рис. 1. По окончании сканирования мышей, все еще находящихся в состоянии общей анестезии, подвергали эвтаназии методом цервикальной дислокации.

Количественное определение концентрации ДПА в опухоли проводили по значениям рентгеноплотности каждого пикселя участка томограммы относительным методом. Учитывая, что абсолютные реконструируемые

Таблица. Основные физико-химические характеристики раствора Ві-ДТПА

\begin{tabular}{|l|c|}
\hline \multicolumn{1}{|c|}{ Параметр } & Значение \\
\hline Брутто-формула & $\mathrm{BiNa}_{2} \mathrm{C}_{14} \mathrm{H}_{18} \mathrm{O}_{10} \mathrm{~N}_{3}$ \\
\hline Молекулярная масса, Дальтон & 643,253 \\
\hline Содержание металла, \% от массы & 34 \\
\hline Концентрация, $\mathrm{M}$ & 0,5 \\
\hline рН раствора & 7,4 \\
\hline Плотность, г/см ${ }^{3}$ при $20^{\circ} \mathrm{C}$ & 1,19 \\
\hline Устойчивость комплекса, logK & 31 \\
\hline
\end{tabular}

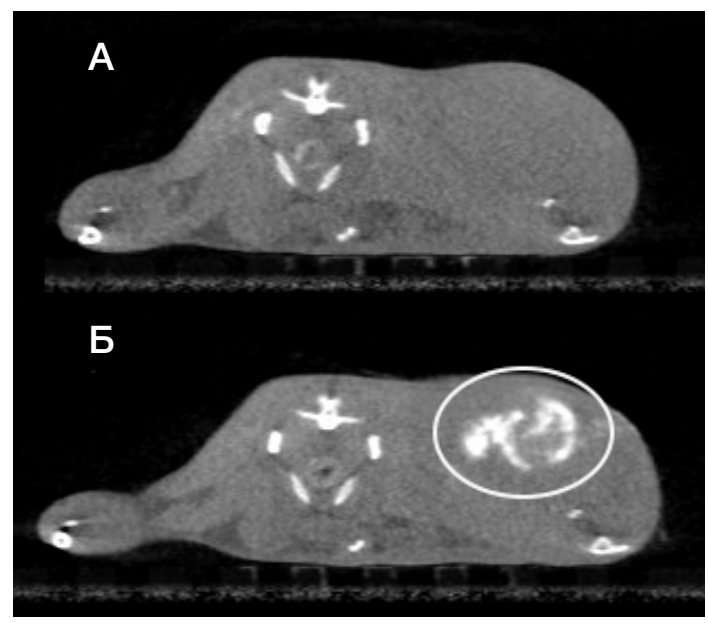

Рис. 1. Томограммы мыши с меланомой B16F10: (А) до интратуморального введения водного раствора Ві-ДТПА в объеме 50 мкл с концентрацией висмута 104,5 мг/мл; (Б) после введения 
значения рентгеноплотности для однородного объекта у сканера IVIS Spectrum CT зависят от номера среза и значительно различаются в центральных областях объекта по сравнению с периферическими [22], для расчетов концентрации ДПА использовали разницу значений рентгеноплотности опухолевых тканей до введения ДПА и после введения. Пересчет значений рентгеноплостности в значения концентрации ДПА осуществляли по градуировочным прямым, построенным по томограммам микропробирок, содержащих растворы с известной концентрацией висмута (рис. 2).

Оценку величины поглощенной дозы для каждого пикселя томограммы опухоли проводили путем определения фактора повышения дозы (ФПД) для соответствующего значения концентрации ДПА, полученной для данного пикселя в каждом исследованном временном интервале. ФПД - это отношение значений поглощенной дозы в объекте в присутствии и в отсутствии ДПА для одних и тех же параметров внешнего облучения, определяемое по формуле:

$$
\text { ФПД }=D_{\text {дПА }}(C) / D_{0}
$$

где $D_{\text {дпн }}(C)$ - поглощенная доза в точке некоторого объекта, содержащая концентрацию ДПА величиной $C$ при облучении рентгеновским излучением с интенсивностью I В данной точке, $D_{0}$ - поглощенная доза в той же точке того же объекта, имеющая концентрацию ДПА, равную нулю $(C=0)$, при облучении рентгеновским излучением той же интенсивности I [23].

Величину ФПД определяли по измеренным значениям концентрации ДПА на основании экспериментальных и расчетных данных [2, 24]. Значения ФПД усредняли по времени на интервале от 0 до 30 мин, что соответствует времени облучения, использованного нами в более ранней работе [15]. Затем воксели опухоли, имеющие одинаковые средние значения ФПД, суммировали для получения дозообъемного распределения. Поскольку в ФЗТ наличие ДПА в опухоли приводит к локальному увеличению поглощенной дозы (т. е. опухоль или ее часть получает дозу излучения, превышающую номинальную, рассчитанную без учета присутствия ДПА), в данной работе мы использовали модисицированный вид кумулятивного дозо-объемного распределения, отличный от применяемого в конвенциональной лучевой терапии. Для характеристики величины и пространственного распределения поглощенной

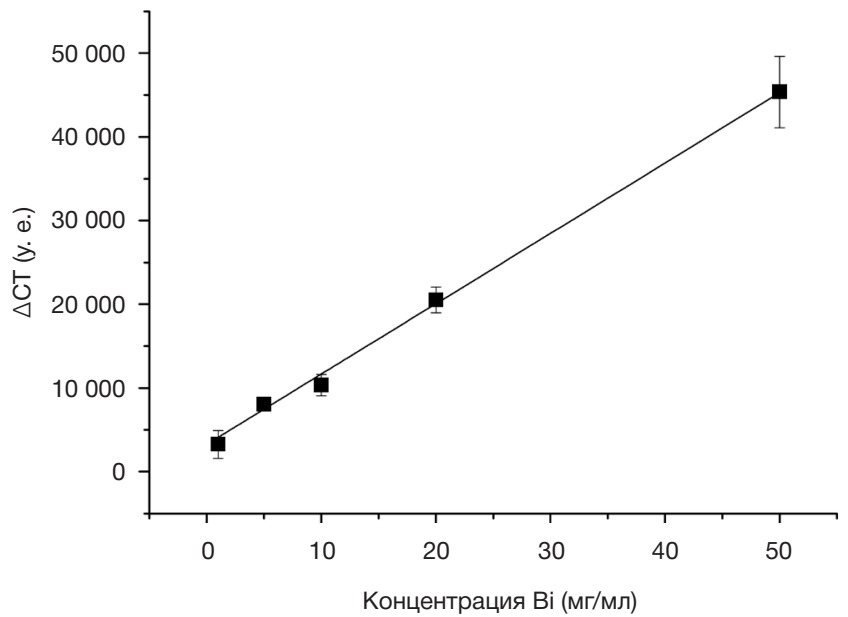

Рис. 2. Градуировочная зависимость изменения рентгеноплотности $(\triangle \mathrm{CT})$ на томограммах микро-КТ сканера IVIS Spectrum СТ от концентрации висмута дозы использовали кумулятивное дозо-объемное распределение, показывающее зависимость ФПД от относительного объема опухоли (в \%). Для каждого значения относительного объема опухоли указывали соответствующую для него минимальную величину ФПД. Данный вид дозо-объемного распределения более информативен для ФЗТ, так как позволяет наглядно оценить роль используемого препарата с ДПА в увеличении поглощенной дозы в органе. Анализ томограмм, расчет ФПД и дозо-объемных распределений проводили в программной среде MATLAB (MathWorks; CША).

\section{РЕЗУЛЬТАТЫ ИССЛЕДОВАНИЯ}

Анализ распределения Ві-ДТПА в опухолевых тканях после однократного интратуморального введения показал, что период полувыведения комплекса из опухоли составляет 3 мин. К 30-й минуте после введения в опухоли остается $4 \%$ от введенного количества (рис. 3).

Объем распределения введенного раствора Ві-ДТПА через 1 мин после введения составил $219 \pm 35$ мм $^{3}$ (24 \pm 1\%) от общего объема опухоли (рис. 4).

До 3-й минуты после введения наблюдается конкурирующее действие процесса распределения раствора Ві-ДТПА в опухолевых тканях и процесса его выведения, в результате чего доля объема опухоли, содержащего ДПА, не изменяется. Далее объем опухоли, содержащий ДПА, уменьшался и к 25-й минуте после введения составил 1-7\% от общего объема опухоли. Полученное модифицированное кумулятивное дозообъемное распределение представлено на рис. 5.

По данным дозо-объемного распределения, в 6\% объема опухоли наблюдали увеличение поглощенной дозы более чем в 2 раза. Дополнительное энерговыделение за счет присутствия ДПА, превышающее номинальную дозу облучения (ФПД > 1), было обнаружено в 29\% объема опухоли.

\section{ОБСУЖДЕНИЕ РЕЗУЛЬТАТОВ}

Полученное дозо-объемное распределение (рис. 5) характеризуется выраженной неоднородностью по объему опухоли. Максимальное рассчитанное значение ФПД в области максимальной концентрации ДПА при облучении в течение 28,5 мин равно 4. Это позволяет при рентгеновском облучении дозой 0,7 Гр/мин обеспечить поглощенную дозу

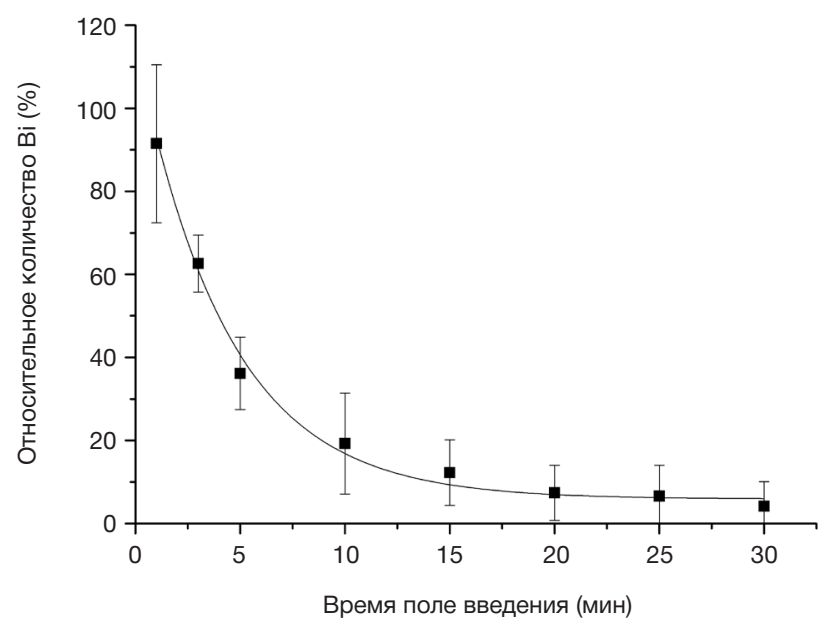

Рис. 3. График зависимости содержания висмута в опухоли относительно введенного количества от времени, прошедшего после введения 


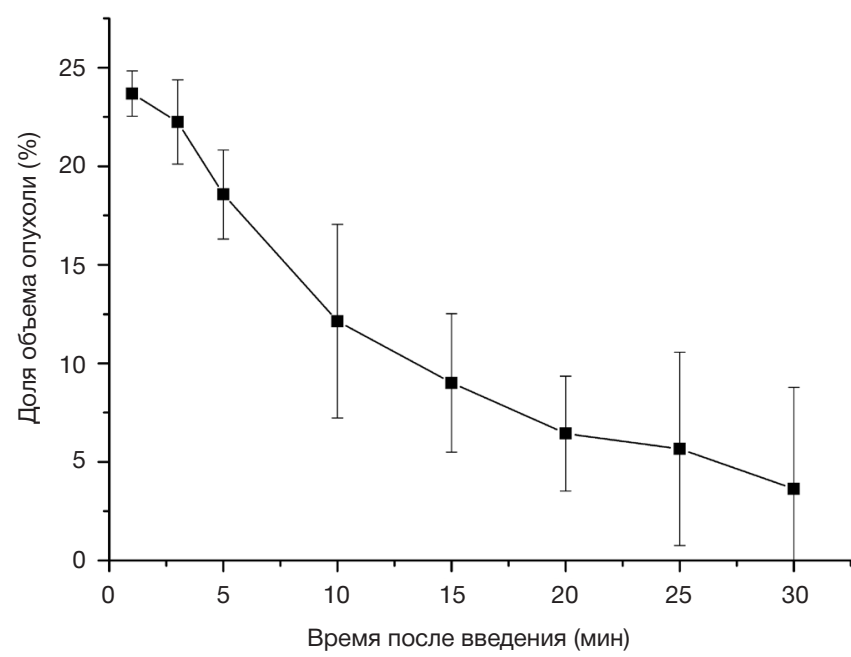

Рис. 4. График зависимости относительного объема опухоли, содержащий ДПА, от времени, прошедшего после введения

величиной 80 Гр. Однако такое значительное увеличение поглощенной дозы было характерно лишь для 0,1\% объема опухоли. Увеличение поглощенной дозы в опухоли в 1,5 и более раза (т. е. поглощенная доза более 30 Гр при той же мощности дозы) за счет присутствия в ней ДПА, наблюдали в 10\% объема опухоли.

С целью дальнейшей оценки максимально возможного времени задержки роста опухоли, облученной с полученным дозо-объемным распределением, были использованы следующие положения:

1) считали, что рецидивирующая после облучения опухоль растет по экспоненциальному закону с временем удвоения $T d$ [25];

2) клетки в опухоли погибают только в результате непосредственного радиационного повреждения;

3) допускали, что в 10\% объема опухоли, получившей дозу 30 Гр, произошла полная гибель всех опухолевых клеток.

Последнее допущение использовали с целью упростить оценку именно максимально возможного времени задержки роста опухоли, что в этой ситуации допустимо: если часть клеток в данном объеме все-таки выживет, то это уменьшит время задержки роста опухоли и не повлияет на величину максимального значения. Тогда, если объем выживших клеток после облучения без введения ДПА принять за $V_{s}$, объем выживших клеток в опухоли с интратуморальным введением ДПА составит $0,9 V_{\text {s. }}$ Если считать, что время удвоения объема опухоли в обоих случаях одинаковое и равно 5 сут. $[17,26]$, а рост

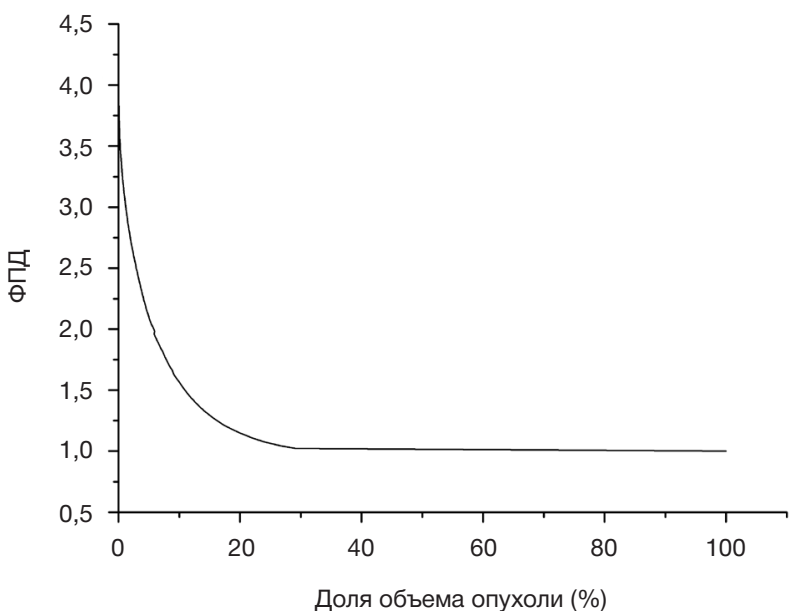

Рис. 5. Модифицированное дозо-объемное распределение для меланомь B16F10 при рентгеновском облучении в течение 28,5 мин с предварительным интратуморальным введением ДПА

объема происходит по экспоненциальному закону, то время задержки роста опухоли за счет интратуморального введения ДПА перед облучением не превысит 0,76 сут.

Однако экспериментальные исследования, опубликованные нами ранее [15], показывают, что такое введение обеспечивает задержку роста опухоли на 10 сут. по сравнению с опухолью, облученной без введения ДПА. Очевидно, что противоопухолевый эффект, наблюдаемый при однократном рентгеновском облучении меланомы B16F10 с интратуморальным введением ДПА, обусловлен не только непосредственным радиационным поражением опухолевых клеток, но и другими механизмами.

\section{ВЫВОДЫ}

Анализ пространственного распределения ДПА и поглощенной дозы в объеме опухоли показал, что однократное интратуморальное введение обеспечивает выраженное неоднородное распределение ДПА и, как следствие, поглощенной дозы в опухоли. Для достижения более однородного распределения целесообразно использовать многократное обкалывание опухоли, которое желательно проводить под контролем средств визуализации (УЗИ, КТ др.). Полученные ранее результаты по противоопухолевому эффекту ФЗТ с интратуморальным введением ДПА [15] ставят вопрос о необходимости изучения механизмов противоопухолевого действия ФЗТ с данным путем введения ДПА.

\section{Литература}

1. Siegel RL, Miller KD, Jemal A. Cancer statistics, 2015. CA Cancer J Clin. 2015 Jan; 65 (1): 5-29.

2. Robar JL, Riccio S, Martin M. Tumour dose enhancement using modified megavoltage pho-ton beams and contrast media. Phys Med Biol. 2002 Jul 21; 47 (14): 305

3. Кулаков В. Н., Липенгольц А. А., Григорьева Е. Ю. Шимановский Н. Л. Препараты для дистанционной бинарной лучевой терапии и их применение при злокачественных новооб-разованиях. Химико-фармацевтический журнал. 2016; 50 (6): 19-25.

4. Roeske JC, Nunez L, Hoggarth M, Labay E, Weichselbaum RR. Characterization of the Theo-rectical Radiation Dose Enhancement from Nanoparticles. Technol Cancer Res Treat. 2007; 6 (5): 395-401.

5. Norman A, Ingram M, Skillen RG, Freshwater DB, Iwamoto KS, Solberg T. X-ray photother-apy for canine brain masses. Radiat Oncol Investig. 1997; 5 (1): 8-14.

6. Miladi I, Alric C, Dufort S, et al. The In Vivo Radiosensitizing Effect of Gold Nanoparticles Based MRI Contrast Agents. Small. 2014; 10 (6): 1116-24.

7. Le Duc G, Miladi I, Alric C, et al. Toward an Image-Guided Microbeam Radiation Therapy Using Gadolinium-Based Nanoparticles. ACS Nano. 2011; 5 (12): 9566-74.

8. Hainfeld JF, Dilmanian FA, Zhong Z, Slatkin DN, Kalef-Ezra JA, Smilowitz HM. Gold nano-particles enhance the radiation therapy of a murine squamous cell carcinoma. Phys Med Biol. 2010; 55 (11): 3045-59. 
9. Hainfeld JF, Slatkin DN, Smilowitz HM. The use of gold nanoparticles to enhance radiothera-py in mice. Phys Med Biol. 2004; 49 (18): 309-15.

10. Hainfeld JF, Smilowitz HM, O'Connor MJ, Dilmanian FA, Slatkin DN. Gold nanoparticle imaging and radiotherapy of brain tumors in mice. Nanomedicine. 2013; 8 (10): 1601-09.

11. Dufort S, Le Duc G, Salomé M, et al. The High Radiosensitizing Efficiency of a Trace of Gadolinium-Based Nanoparticles in Tumors. Sci Rep. 2016; 6 (June): 1-8.

12. Komatsu T, Nakamura K, Okumura Y, Konishi K. Optimal method of gold nanoparticle ad-ministration in melanoma bearing mice. Exp Ther Med. 2018 Jan 12; 15 (3): 2994-9.

13. Rousseau J, Boudou $C$, Estève $F$, Elleaume $H$. ConvectionEnhanced Delivery of an lodine Tracer Into Rat Brain for Synchrotron Stereotactic Radiotherapy. Int J Radiat Oncol. 2007 Jul; 68 (3): 943-51.

14. Mello RS, Callisen H, Winter J, Kagan a R, Norman A. Radiation dose enhancement in tu-mors with iodine. Med Phys [Internet]. 1983 Jan; 10 (1): 75-8.

15. Липенгольц А. А., Черепанов А. А., Кулаков В. Н., Григорьева Е. Ю., Меркулова И. Б., Шейно И. Н. Сравнение противоопухолевой эфффективности висмута и гадолиния как дозоповышающих агентов в препаратах для фотонзахватной терапии. Химико-фармацевтический журнал. 2017; 51 (9): 34-7.

16. Черепанов А. А., Липенгольц А. А., Насонова Т. А., Добрынина О. А., Кулаков В. Н., Шейно И. Н., и др. Увеличение противоопухолевого эффекта рентгеновского облучения при помощи гадолиний-содержащего препарата на примере мышей с трансплантированной меланомой B16F10. Медицинская физика. 2014; (3): 66-9.

17. Maggiorella L, Barouch G, Devaux C, Pottier A, Deutsch E, Bourhis $\mathrm{J}$, et al. Nanoscale radio-therapy with hafnium oxide nanoparticles. Futur Oncol. 2012; 8 (9): 1167-81.

18. Bonvalot S, Le Pechoux C, De Baere T, Kantor G, Buy X, Stoeckle E, et al. First-in-Human Study Testing a New Radioenhancer Using Nanoparticles (NBTXR3) Activated by Radiation Therapy in Patients with Locally Advanced Soft Tissue Sarcomas. Clin Cancer Res. 2017 Feb 15; 23 (4): 908-17.
19. Hainfeld JF, O'Connor MJ, Dilmanian FA, Slatkin DN, Adams DJ, Smilowitz HM. Micro-CT enables microlocalisation and quantification of Her2-targeted gold nanoparticles within tu-mour regions. Br J Radiol. 2011; 84 (1002): 526-33. DOl:10.1259/ bjr/42612922.

20. Le Duc G, Corde S, Charvet A-M, Elleaume H, Farion $R$, Le Bas J-F, et al. In Vivo Meas-urement of Gadolinium Concentration in a Rat Glioma Model by Monochromatic Quantitative Computed Tomography. Invest Radiol. 2004 Jul; 39 (7): 385-93.

21. Липенгольц А. А., Будаева Ю. А., Блайкнер М., Черепанов А. А., Меньков М. А., Кулаков В. Н., и др. Количественное определение йода при помощи рентгеновской компьютерной томографии для дозиметрического обеспечения фотонзахватной терапии. Вестник РГМУ. 2016; (6): 16-20.

22. Pervova W, Lipengolts AA, Cherepanov AA, Abakumov MA. Study of iodine, gadolinium and bismuth quantification possibility with micro-CT IVIS spectrumct in vivo imaging system. J Phys Conf Ser. 2017; 784 (1): 12043.

23. Воробьева Е. С., Липенгольц А. А., Черепанов А. А., Григорьева Е. Ю., Нечкина И. Н., Калыгина Н. С., и др. Возможность проведения фотон-захватной терапии с использованием 6 МВ фотонного излучения. Вестник РГМУ. 2017; (4): 63-7.

24. Черепанов А. А., Липенгольц А. А., Воробьева Е. С., Кулаков В. Н., Климанов В. А., Григорьева Е. Ю. Исследование увеличения энерговыделения в среде за счет присутствия тяжелого элемента с использованием дозиметра Фрике. Медицинская физика. 2016; 72 (4): 38-41.

25. Bristow RG, Hill RP. Comparison between in vitro radiosensitivity and in vivo radioresponse in murine tumor cell lines $\mathrm{I}$ : in vivo radioresponse following fractionated treatment and in vitro/in vivo correlations. Int J Radiat Oncol. 1990 Feb; 18 (2): 331-45.

26. Le UM, Kaurin DGL, Sloat BR, Yanasarn N, Cui Z. Localized irradiation of tumors prior to synthetic dsRNA therapy enhanced the resultant anti-tumor activity. Radiother Oncol. 2009 Feb; 90 (2): 273-9.

\section{References}

1. Siegel RL, Miller KD, Jemal A. Cancer statistics, 2015. CA Cancer J Clin. 2015 Jan; 65 (1): 5-29.

2. Robar JL, Riccio S, Martin M. Tumour dose enhancement using modified megavoltage pho-ton beams and contrast media. Phys Med Biol. 2002 Jul 21; 47 (14): 305.

3. Kulakov VN, Lipengolts AA, Grigoreva EY, Shimanovskii NL. Pharmaceuticals for Binary Radiotherapy and Their Use for Treatment of Malignancies (A Review). Pharm Chem J. 2016 Sep 8; 50 (6): 19-25.

4. Roeske JC, Nunez L, Hoggarth M, Labay E, Weichselbaum RR. Characterization of the Theo-rectical Radiation Dose Enhancement from Nanoparticles. Technol Cancer Res Treat. 2007; 6 (5): 395-401.

5. Norman A, Ingram M, Skillen RG, Freshwater DB, Iwamoto KS, Solberg T. X-ray photother-apy for canine brain masses. Radiat Oncol Investig. 1997; 5 (1): 8-14.

6. Miladi I, Alric C, Dufort S, et al. The In Vivo Radiosensitizing Effect of Gold Nanoparticles Based MRI Contrast Agents. Small. 2014; 10 (6): 1116-24.

7. Le Duc G, Miladi I, Alric C, et al. Toward an Image-Guided Microbeam Radiation Therapy Using Gadolinium-Based Nanoparticles. ACS Nano. 2011; 5 (12): 9566-74.

8. Hainfeld JF, Dilmanian FA, Zhong Z, Slatkin DN, Kalef-Ezra JA, Smilowitz HM. Gold nano-particles enhance the radiation therapy of a murine squamous cell carcinoma. Phys Med Biol. 2010; 55 (11): 3045-59.

9. Hainfeld JF, Slatkin DN, Smilowitz HM. The use of gold nanoparticles to enhance radiothera-py in mice. Phys Med Biol. 2004; 49 (18): 309-15
10. Hainfeld JF, Smilowitz HM, O'Connor MJ, Dilmanian FA Slatkin DN. Gold nanoparticle imaging and radiotherapy of brain tumors in mice. Nanomedicine. 2013; 8 (10): 1601-09.

11. Dufort S, Le Duc G, Salomé M, et al. The High Radiosensitizing Efficiency of a Trace of Gadolinium-Based Nanoparticles in Tumors. Sci Rep. 2016; 6 (June): 1-8.

12. Komatsu T, Nakamura K, Okumura $Y$, Konishi K. Optimal method of gold nanoparticle ad-ministration in melanoma bearing mice. Exp Ther Med. 2018 Jan 12; 15 (3): 2994-9.

13. Rousseau J, Boudou C, Estève F, Elleaume H. Convection-Enhanced Delivery of an lodine Tracer Into Rat Brain for Synchrotron Stereotactic Radiotherapy. Int J Radiat Oncol. 2007 Jul; 68 (3): 943-51.

14. Mello RS, Callisen H, Winter J, Kagan a R, Norman A. Radiation dose enhancement in tu-mors with iodine. Med Phys [Internet]. 1983 Jan; 10 (1): 75-8.

15. Lipengolts AA, Cherepanov AA, Kulakov VN, Grigoreva EY, Merkulova IB, Sheino IN. Comparison of the Antitumor Efficacy of Bismuth and Gadolinium as Dose-Enhancing Agents in Formulations for Photon Capture Therapy. Pharm Chem J. 2017 Dec 5; 51 (9): 783-6.

16. Cherepanov AA, Lipengolts AA, Nasonova TA, Dobrynina OA, Kulakov VN, Sheino IN et al. Increasing of antineoplastic effect of $\mathrm{x}$-ray irradiation in mice with transplanted melanoma B16F10 by use of gadolinium containing drug. Meditsinskaya fizika. 2014; (3): 66-9. (in Russian)

17. Maggiorella L, Barouch G, Devaux C, Pottier A, Deutsch E, Bourhis $\mathrm{J}$, et al. Nanoscale radio-therapy with hafnium oxide nanoparticles. Futur Oncol. 2012; 8 (9): 1167-81. 
18. Bonvalot S, Le Pechoux C, De Baere T, Kantor G, Buy X, Stoeckle E, et al. First-in-Human Study Testing a New Radioenhancer Using Nanoparticles (NBTXR3) Activated by Radiation Therapy in Patients with Locally Advanced Soft Tissue Sarcomas. Clin Cancer Res. 2017 Feb 15; 23 (4): 908-17.

19. Hainfeld JF, O'Connor MJ, Dilmanian FA, Slatkin DN, Adams DJ, Smilowitz HM. Micro-CT enables microlocalisation and quantification of Her2-targeted gold nanoparticles within tu-mour regions. Br J Radiol. 2011; 84 (1002): 526-33. DOI:10.1259/ bjr/42612922.

20. Le Duc G, Corde S, Charvet A-M, Elleaume H, Farion R, Le Bas J-F, et al. In Vivo Meas-urement of Gadolinium Concentration in a Rat Glioma Model by Monochromatic Quantitative Computed Tomography. Invest Radiol. 2004 Jul; 39 (7): 385-93.

21. Lipengolts $A A$, Budaeva JA, Blaickner $M$, Cherepanov $A A$, Menkov MA, Kulakov VN, et al. lodine quantification with computed tomography for the purpose of dose assessment in contrast enhanced radiotherapy. Bull Russ State Med Univ. 2016; (6): 16-9.
22. Pervova W, Lipengolts AA, Cherepanov AA, Abakumov MA. Study of iodine, gadolinium and bismuth quantification possibility with micro-CT IVIS spectrumct in vivo imaging system. J Phys Conf Ser. 2017; 784 (1): 12043.

23. Vorobyeva ES, Lipengolts AA, Cherepanov AA, Grigorieva EY, Nechkina IN, Kalygina NS, et al. Feasibility of using 6 MV photon beams in contrast-enhanced radiotherapy. Bull Russ State Med Univ. 2017; (4): 57-61.

24. Cherepanov AA, Lipengolts AA, Vorobyeva ES, Kulakov VN, Klimanov VA, Grigorieva EYu. Experimental study of $x$-rays absorbed dose increase in medium containing high-Z element using Fricke dosimeter. Meditsinskaya fizika. 2016; 72 (4): 38-41. (in Russian)

25. Bristow RG, Hill RP. Comparison between in vitro radiosensitivity and in vivo radioresponse in murine tumor cell lines II: in vivo radioresponse following fractionated treatment and in vitro/in vivo correlations. Int J Radiat Oncol. 1990 Feb; 18 (2): 331-45.

26. Le UM, Kaurin DGL, Sloat BR, Yanasarn N, Cui Z. Localized irradiation of tumors prior to synthetic dsRNA therapy enhanced the resultant anti-tumor activity. Radiother Oncol. 2009 Feb; 90 (2): 273-9. 